\title{
Manipulability Optimization Control of a Serial Redundant Robot for Robot-assisted Minimally Invasive Surgery
}

\author{
Hang $\mathrm{Su}^{1}$, Shuai $\mathrm{Li}^{2}$, Jagadesh Manivannan ${ }^{1}$, Luca Bascetta ${ }^{1}$, Giancarlo Ferrigno ${ }^{1}$ and Elena De Momi ${ }^{1}$
}

\begin{abstract}
This paper proposes a manipulability optimization control of a 7-DoF robot manipulator for Robot-Assisted Minimally Invasive Surgery (RAMIS), which at the same time guarantees a Remote Center of Motion (RCM). The first degree of redundancy of the manipulator is used to achieve an RCM constraint, the second one is adopted for manipulability optimization. A hierarchical operational space formulation is introduced to integrate all the control components, including a Cartesian compliance control involving the main surgical task, a first null-space controller for the RCM constraint, and a second null-space controller for manipulability optimization. Experiments with virtual surgical tasks, in an augmented reality environment, were performed to validate the proposed control strategy using the KUKA LWR4+. The results demonstrate that end-effector accuracy and RCM constraint can be guaranteed, along with improving the manipulability of the surgical tip.
\end{abstract}

\section{INTRODUCTION}

Robot-assisted surgery has been more popular over the recent years for the advantages it can offer, like greater surgical precision, increased range of motion, improved dexterity and enhanced visualization for surgeons [1]. In the traditional Minimally Invasive Surgery, the surgical tip must go through small incisions on the patient's abdominal wall. Each small incision produces a kinematic constraint, commonly known as the Remote Center of Motion (RCM) constraint [2]. In general, the RCM constraint can be active or passive, where the passive constraint is maintained mechanically, while the active method is known to be achieved with a software controller [3], and is more popular in the nonclinical research, since it is cost-less and provides a flexible task space.

Different approaches have been adopted to derive a generic formalization of the RCM as a kinematic constraint [2] and to actively enforce it. In particular, Ortmaier et al. [4] proposed an inverse kinematic control to enforce the motion constraint preventing any force to be exerted on the trocar during robotic surgery. However, the adoption of position control in a surgical operation may cause, in case of rigid interaction, high contact forces [5], [6]. For this reason, From et al. [7], while addressing the RCM constraint in task space, introduced Cartesian impedance control to perform

\footnotetext{
*This work was supported by the European Commission Horizon 2020 research and innovation program, under the project SMARTsurg, grant agreement No. 732515.

${ }^{1}$ Hang Su, Jagadesh Manivannan, Luca Bascetta, Giancarlo Ferrigno and Elena De Momi are with the Dipartimento di Elettronica, Informazione e Bioingegneria, Politecnico di Milano, 20133, Milano, Italy. \{hang.su, jagadesh.manivannan, luca.bascetta, giancarlo.ferrigno, elena.demomi\}@polimi.it

${ }^{2}$ Shuai Li is with the Department of Computing, The Hong Kong Polytechnic University, Kowloon, Hong Kong. shuaili@polyu . edu . hk
}

the tasks safely. A step further in enforcing the RCM constraint was performed by Sandoval et al. [3], exploiting the redundancy. In fact, they proposed an improved dynamic control approach that takes advantage of task redundancy for the RCM constraint. Similarly, in [8] an adaptive decoupling controller exploiting task redundancy was proposed, as well.

Besides fulfilling the RCM constraint, redundancy can also be exploited to achieve other additional tasks, e.g., obstacle avoidance [9], human-like behavior [10], and manipulability optimization [11]. In particular, manipulability optimization [12], [13], [14] of a surgical tool tip plays an important role in robot assisted surgery, as it can provide maximum distance from singularities, more flexible motion, wider operational space, etc., and local optimization methods, e.g., based on gradient projection as in [15] or on singular value decomposition as in [16], has been already introduced.

In this paper, a novel manipulability optimization control of a serial redundant robot for real-time robot-assisted Minimally Invasive Surgery has been proposed. The method exploits the hierarchical operational space formulation [17], integrating three parts: a Cartesian compliance control strategy for the main surgical tracking task, a first null-space controller to enforce the RCM constraint, and a second nullspace controller for manipulability optimization by way of a constrained quadratic programming approach.

It must be noticed that the proposed methodology represents an improvement with respect to the RCM solution introduced in [3], and combines an efficient on-line manipulability optimization [11] into a single controller.

Finally, experiments using a 7-DoF serial robot KUKA LWR4+ and considering a virtual surgical task tracking have been performed to validate the proposed control method.

The paper is organized as follows. Section II describes the proposed robot assisted MIS system. The dynamic model of the redundant manipulator and the corresponding control methodology are presented in Section III. In Section IV the performance of the proposed control scheme is evaluated using the KUKA LWR4+ robot, and conclusions are drawn in Section V.

\section{System Description}

An overview of the developed robot assisted MIS system is shown in Figure 1. The system is composed of:

- a KUKA LWR4+ redundant robot, that is torquecontrolled through the Fast Research Interface (FRI), providing a direct real-time access to the robot controller at $500 \mathrm{~Hz}[18]$; 
- an HD endoscopic camera and an ArUco marker board [19], that are used for virtual surgical task [20], [21] tracking in an augmented reality environment;

- a 6-axis M8128C6 force sensor, adopted to measure the interaction force between the surgical tip and the abdominal wall.

The control system has been developed using OROCOS ${ }^{1}$, with a real-time Xenomai-patched Linux kernel, and using ROS $^{2}$ Kinetic under Ubuntu [20].

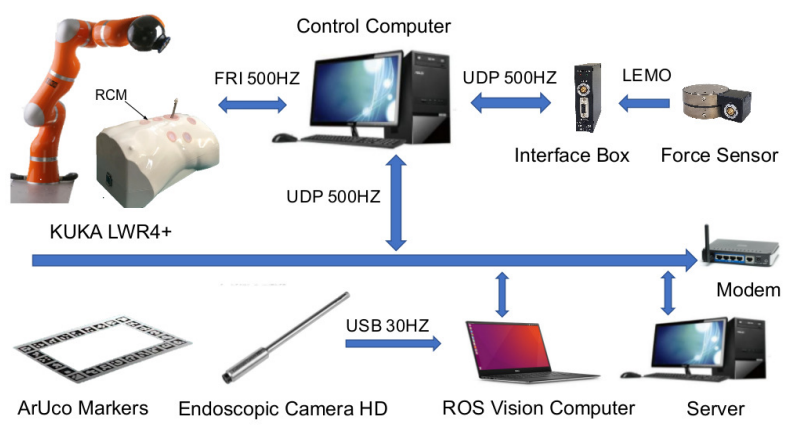

Fig. 1. Overview of the robot assisted surgical MIS system.

\section{Control methodology}

The control methodology here proposed aims at allowing a redundant robot to autonomously perform a surgical task, tracking a desired trajectory of the surgical tool tip generated by a surgeon, while ensuring the required level of safety by way of a task space impedance controller, and using the redundant degrees of freedom to enforce an RCM constraint and optimize the manipulability. The robot model has been discussed in [20], [21]. This Section introduces the proposed control solution, based on the hierarchical control formulation [17].

\section{A. Hierarchical operational space formulation}

The hierarchical control architecture (Figure 2), formulated in [17], is here composed of three main functions:

- a Cartesian compliance control strategy, accounting for the main surgical tracking task;

- a first null-space controller, to enforce the RCM constraint;

- a second null-space controller, to optimize the robot manipulability.

Each of these functions is described in the remaining of this Section, while the stability analysis of the overall system has been discussed in [22], [23].

\section{B. Autotracking surgical task implementation}

The main task for the MIS robot is tracking the desired Cartesian trajectory generated by the surgeon [24], while guaranteeing safety by way of a Cartesian impedance

\footnotetext{
${ }^{1}$ Open Robotic Control Software, http://www.orocos.org/

${ }^{2}$ Robot Operating System, http://www.ros.org/
}

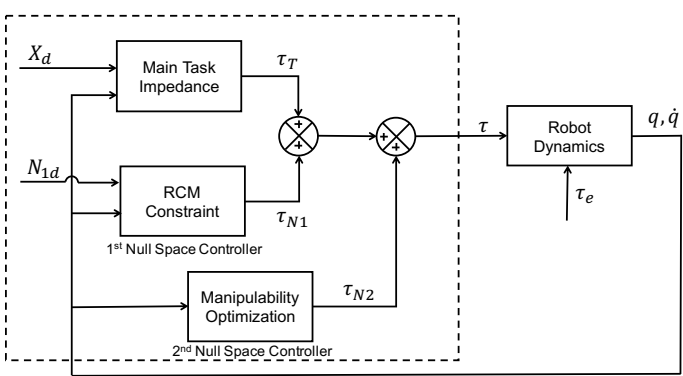

Fig. 2. Block diagram representing the hierarchical control architecture.

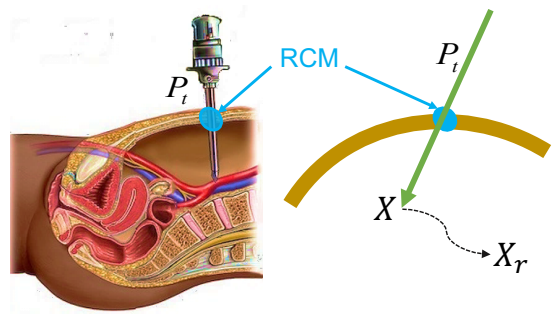

Fig. 3. Robot-assisted MIS surgical scene: during surgery, the tool-tip must go through the trocar position $P_{t}$, representing the RCM constraint. $X$ and $X_{r}$ are the actual and desired Cartesian position inside the abdomen.

controller.

First of all, to guarantee the required smoothness to the surgical tool tip motion and avoid the trajectory generated by the surgeon exceeds the robot acceleration and velocity constraints, an interpolation function is introduced. In particular, the surgeon trajectory is interpolated using a $5^{\text {th }}$ order polynomial in Cartesian space [25], adapting the trajectory duration in such a way that the maximum Cartesian acceleration and velocity constraints are satisfied. Then a Cartesian space compliance control term [20], $\boldsymbol{\tau}_{T} \in \mathbb{R}^{7}$, can be introduced to achieve the main surgical tasks.

\section{RCM constraint in the first null-space}

In the case of auto-tracking of a surgical task, three out of the seven robot degrees of freedom are exploited, the other four degrees of freedom remain as redundancy. Part of these redundant degrees of freedom are used here by the first nullspace controller to fulfil the RCM constraint.

Figure 3 shows that to guarantee the tool always enters the patient body through the trocar point $\boldsymbol{P}_{t}$, without influencing the main surgical task, i.e., acting in the null space of the surgical task. Hence, the "wrist" joint has to track a circular movement [21] around the end-effector from its actual position $\boldsymbol{N}_{1}$ to the desired position $\boldsymbol{N}_{1 d}$. A control action $\boldsymbol{F}_{N 1}$ is proposed in [21] using surgical task redundancy to enforce this constraint and to reduce the "wrist" tracking error $\boldsymbol{e}_{N 1}=\left\|\boldsymbol{N}_{1}-\boldsymbol{N}_{1 d}\right\|$. Projecting this action into the null-space of the surgical task, the first null-space controller of the surgical tip can be introduced as:

$$
\boldsymbol{\tau}_{N 1}=\left(\boldsymbol{I}-\boldsymbol{J}_{T}^{T}(\boldsymbol{q}) \boldsymbol{J}_{T}(\boldsymbol{q})\right) \boldsymbol{J}_{W}^{T}(\boldsymbol{q}) \boldsymbol{F}_{N 1}
$$


where $\boldsymbol{J}_{W}(q) \in \mathbb{R}^{3 \times 5}$ is the Jacobian matrix from the base frame to the robot wrist. Furthermore, error $e_{N 1} \in \mathbb{R}$ can be represented using the following distance [20]

$$
d=\left\|\left(\boldsymbol{P}_{t}-\boldsymbol{X}\right) \times \hat{\boldsymbol{u}}_{c}\right\|
$$

where $\hat{\boldsymbol{u}}_{c} \in \mathbb{R}^{3}$ is the actual surgical tip direction vector. In fact, it can be easily shown that $\left\|\boldsymbol{e}_{N}\right\|$ is proportional to the RCM constraint error $d$, i.e., $d=\lambda\left\|\boldsymbol{e}_{N 1}\right\|, \lambda \in(0,1]$, and it can be thus expressed as

$$
\boldsymbol{e}_{N 1}=\frac{\left\|\left(\boldsymbol{P}_{t}-\boldsymbol{X}\right) \times \hat{\boldsymbol{u}}_{c}\right\|}{\lambda}
$$

where $\boldsymbol{P}_{t}, \boldsymbol{X}$ and $\hat{\boldsymbol{u}}_{c}$ are all known. In general, $\lambda$ varies according to the tip position during the operation. In this paper, we choose $\lambda$ as a constant, but we resolve its timedependency introducing manipulability optimization.

\section{Manipulability optimization in the second null-space}

The additional redundant degrees of freedom, corresponding to the "wrist" null-space, are used to optimize the manipulability in the way shown in Figure 4, i.e., the positions of end-effector and "wrist" are fixed, while the other joints move to achieve manipulability optimization.

As it is well-known the manipulability measure gives a scalar representation of the gain between joint velocities $\dot{\boldsymbol{q}}$ and task velocities $\dot{\boldsymbol{X}}$, and, consequently, measures the ability of the robot to move its end-effector, or, equivalently, the distance from a singular configuration, i.e., a configuration $\boldsymbol{q}$ for which the Jacobian is rank deficient. The manipulability measure depends on the Jacobian matrix and is given by:

$$
\mu=\sqrt{\operatorname{det}\left(\boldsymbol{J}_{T}(\boldsymbol{q}) \boldsymbol{J}_{T}^{T}(\boldsymbol{q})\right)}
$$

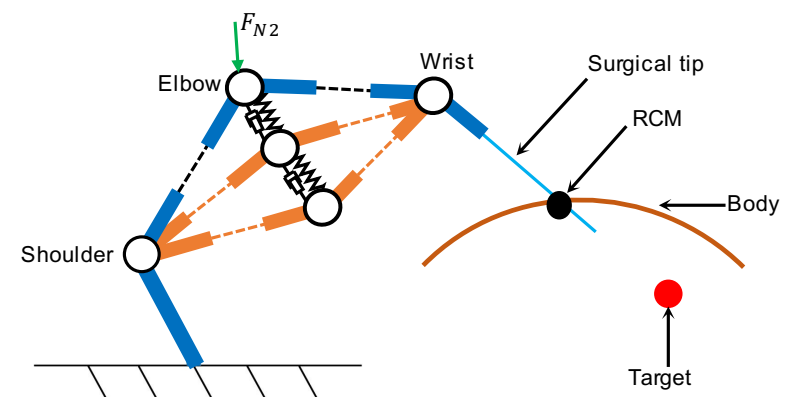

Fig. 4. Achieving manipulability optimization with second null-space of the controller.

1) Optimality formulation: For a redundant manipulator with a desired workspace task $\boldsymbol{X}_{d}$ and a RCM constraint $\boldsymbol{N}_{1 d}$, the manipulability optimization problem can be formulated as [11]:

$$
\begin{aligned}
\min _{\boldsymbol{q} \in \mathbb{R}^{7}} & -\mu \\
\text { s.t. } & \boldsymbol{X}_{d}=f_{1}(\boldsymbol{q}) \\
& \boldsymbol{N}_{1 d}=f_{2}(\boldsymbol{q})
\end{aligned}
$$

where $f_{1}(\boldsymbol{q})$ and $f_{2}(\boldsymbol{q})$ are the forward kinematic functions of the end-effector and of the "wrist", respectively. The two equality constraints has been introduced to guarantee that the optimization of the manipulability index does not affect tracking of the desired end-effector trajectory (first equality constraint) and fulfilment of RCM constraint (second equality constraint).

The previous optimization problem is characterized by a cost function that is usually non-convex, and by nonlinear equality constraints, and it thus represents a challenging problem. In order to address the non-convexity of the cost function we can reformulate the optimization problem as follows:

$$
\begin{array}{rc}
\min _{\boldsymbol{q}, \dot{\boldsymbol{q}} \in \mathbb{R}^{7}} & -\dot{\boldsymbol{q}}^{T} \frac{\partial \mu}{\partial \boldsymbol{q}} \\
\text { s.t. } & \boldsymbol{X}_{d}=f_{1}(\boldsymbol{q}) \\
& \boldsymbol{N}_{1 d}=f_{2}(\boldsymbol{q})
\end{array}
$$

On the other side, a way to handle the two nonlinear equality constraints is introduced in Section III-D.2.

Furthermore, during surgical task execution joint velocities and joint angles cannot exceed kinematic limitations, as this might cause problems such as damages and harming the patient. The convex sets of admissible joint positions and velocities can be introduced as follows:

$$
\begin{aligned}
& \Omega_{\boldsymbol{q}}=\left\{q_{i} \in \mathbb{R} \mid \underline{q}_{i} \leq q_{i} \leq \bar{q}_{i}, i=1,2, \ldots, 7\right\} \\
& \Omega_{\dot{\boldsymbol{q}}}=\left\{\dot{q}_{i} \in \mathbb{R} \mid \underline{\dot{q}}_{i} \leq \dot{q}_{i} \leq \dot{\bar{q}}_{i}, i=1,2, \ldots, 7\right\}
\end{aligned}
$$

where $\dot{q}_{i}$ and $\dot{\bar{q}}_{i}$ are lower and upper bounds on joint velocities, respectively, and $\underline{q}_{i}$ and $\bar{q}_{i}$ are lower and upper bounds on joint angles, respectively.

In order to express these two constraints as a single constraint on joint positions and velocities, the constraint on joint positions can be reformulated [11] as follows:

$$
\begin{aligned}
& \Omega_{\boldsymbol{q}}=\left\{\dot{q}_{i} \in \mathbb{R} \mid\right. \\
& \left.\quad-k_{0}\left(q_{i}-\underline{q}_{i}\right) \leq \dot{q}_{i} \leq-k_{0}\left(q_{i}-\bar{q}_{i}\right), i=1,2, \ldots, 7\right\}
\end{aligned}
$$

where $k_{0}$ is a positive constant, and the two constraints can be rewritten as:

$$
\begin{aligned}
\Omega_{\boldsymbol{q}, \dot{\boldsymbol{q}}}=\left\{\dot{q}_{i}\right. & \in \mathbb{R} \mid \max \left(\underline{\dot{q}}_{i},-k_{0}\left(q_{i}-\dot{q}_{i}\right)\right) \leq \dot{q}_{i} \\
& \left.\leq \min \left(\bar{q}_{i},-k_{0}\left(q_{i}-\bar{q}_{i}\right)\right), i=1,2, \ldots, 7\right\}
\end{aligned}
$$

Finally, the manipulability optimization problem, including all the aforementioned constraints, can be formulated as:

$$
\begin{array}{rc}
\min _{\boldsymbol{q}, \boldsymbol{q} \in \mathbb{R}^{7}} & -\dot{\boldsymbol{q}}^{T} \frac{\partial \mu}{\partial \boldsymbol{q}} \\
\text { s.t. } & \boldsymbol{X}_{d}=f_{1}(\boldsymbol{q}) \\
& \boldsymbol{N}_{1 d}=f_{2}(\boldsymbol{q}) \\
\dot{\boldsymbol{q}} & \in \Omega_{\boldsymbol{q}, \dot{\boldsymbol{q}}}
\end{array}
$$

2) Reformulation as a constrained quadratic program: Problem (1) is now reformulated as a constrained quadratic program, in a way that it can be online solved using a RNN as reported in [11]. 
First of all, the two constraints ensuring desired endeffector trajectory tracking and fulfilment of RCM constraint are recast into constraints at velocity level, as follows:

$$
\boldsymbol{J}_{T}(\boldsymbol{q}) \dot{\boldsymbol{q}}-\dot{\boldsymbol{X}}_{d}=0 \quad \boldsymbol{J}_{W}(\boldsymbol{q}) \dot{\boldsymbol{q}}-\dot{\boldsymbol{N}}_{1 d}=0
$$

The equivalence between the two sets of constraints is guaranteed by the fact that the trajectory interpolation function introduced in Section III-B ensures that the initial values satisfy conditions $\boldsymbol{X}_{d_{0}}=f_{1}\left(\boldsymbol{q}_{0}\right), \boldsymbol{N}_{1 d_{0}}=f_{2}\left(\boldsymbol{q}_{0}\right)$.

Furthermore, in order to guarantee that the problem is convex and compliant with the formulation proposed in [11], the objective function is augmented including the constraints as follows:

$$
\frac{c_{0}}{2}\left\|\boldsymbol{J}_{T} \dot{\boldsymbol{q}}-\dot{\boldsymbol{X}}_{d}\right\|^{2}+\frac{c_{1}}{2}\left\|\boldsymbol{J}_{W} \dot{\boldsymbol{q}}-\dot{\boldsymbol{N}}_{1 d}\right\|^{2}-c_{2} \dot{\boldsymbol{q}}^{T} \frac{\partial \mu}{\partial \boldsymbol{q}}
$$

where $c_{0}, c_{1}$, and $c_{2}$ are positive constants.

The optimization problem in (1) can be thus reformulated as:

$$
\begin{array}{cl}
\min _{\boldsymbol{q}, \dot{\boldsymbol{q}}} & \frac{c_{0}}{2}\left\|\boldsymbol{J}_{T} \dot{\boldsymbol{q}}-\dot{\boldsymbol{X}}_{d}\right\|^{2}+\frac{c_{1}}{2}\left\|\boldsymbol{J}_{W} \dot{\boldsymbol{q}}-\dot{\boldsymbol{N}}_{1 d}\right\|^{2}-c_{2} \dot{\boldsymbol{q}}^{T} \frac{\partial \mu}{\partial \boldsymbol{q}} \\
\text { s.t. } & \boldsymbol{J}_{T} \dot{q}-\dot{\boldsymbol{X}}_{d}=0 \\
& \boldsymbol{J}_{W} \dot{q}-\dot{\boldsymbol{N}}_{1 d}=0 \\
& \dot{\boldsymbol{q}} \in \Omega_{\boldsymbol{q}, \dot{\boldsymbol{q}}}
\end{array}
$$

and converted into an unconstrained optimization problem by defining the following Lagrange function:

$$
\begin{array}{r}
L\left(\dot{\boldsymbol{q}}, \boldsymbol{\lambda}_{1}, \boldsymbol{\lambda}_{2}\right)=\frac{c_{0}}{2}\left\|\boldsymbol{J}_{T} \dot{\boldsymbol{q}}-\dot{\boldsymbol{X}}_{d}\right\|^{2}+\frac{c_{1}}{2}\left\|\boldsymbol{J}_{W} \dot{\boldsymbol{q}}-\dot{\boldsymbol{N}}_{1 d}\right\|^{2} \\
-c_{2} \dot{\boldsymbol{q}}^{T} \frac{\partial \mu}{\partial \boldsymbol{q}}+\boldsymbol{\lambda}_{1}^{T}\left(\boldsymbol{J}_{T} \dot{\boldsymbol{q}}-\dot{\boldsymbol{X}}_{d}\right)+\boldsymbol{\lambda}_{2}^{T}\left(\boldsymbol{J}_{W} \dot{\boldsymbol{q}}-\dot{\boldsymbol{N}}_{1 d}\right)
\end{array}
$$

The solution of the unconstrained problem has to satisfy the following Karush-Kuhn-Tucker condition:

$$
-\frac{\partial L}{\partial \dot{\boldsymbol{q}}} \in \boldsymbol{N}_{\Omega_{\boldsymbol{q}, \dot{\boldsymbol{q}}}}(\dot{\boldsymbol{q}})
$$

where $\boldsymbol{N}_{\Omega_{\boldsymbol{q}, \dot{\boldsymbol{q}}}}(\dot{\boldsymbol{q}})$ represents the normal cone of set $\Omega_{\boldsymbol{q}, \dot{\boldsymbol{q}}}$. Introducing a projection operator defined as [11]:

$$
\dot{\boldsymbol{q}}=\boldsymbol{P}_{\Omega_{\boldsymbol{q}, \dot{\boldsymbol{q}}}}\left(\dot{\boldsymbol{q}}-\frac{\partial L}{\partial \dot{\boldsymbol{q}}}\right)
$$

this can be equivalently written as:

$$
\begin{aligned}
\ddot{\boldsymbol{q}}_{d} & =-\dot{\boldsymbol{q}}+\boldsymbol{P}_{\Omega_{\boldsymbol{q}, \dot{\boldsymbol{q}}}}\left(\dot{\boldsymbol{q}}-k \frac{\partial L}{\partial \dot{\boldsymbol{q}}}\right) \\
\dot{\boldsymbol{\lambda}}_{1} & =k \frac{\partial L}{\partial \boldsymbol{\lambda}_{1}} \\
\dot{\boldsymbol{\lambda}}_{2} & =k \frac{\partial L}{\partial \boldsymbol{\lambda}_{2}}
\end{aligned}
$$

and, introducing the expressions of the partial derivatives, as:

$$
\begin{aligned}
\ddot{\boldsymbol{q}}_{d}= & -\dot{\boldsymbol{q}}+\boldsymbol{P}_{\Omega_{\boldsymbol{q}, \dot{\boldsymbol{q}}}}\left(\dot{\boldsymbol{q}}+k\left(c_{2} \frac{\partial \mu}{\partial \boldsymbol{q}}-c_{0} \boldsymbol{J}_{T}^{T}\left(\boldsymbol{J}_{T} \dot{\boldsymbol{q}}-\dot{\boldsymbol{X}}_{d}\right)\right.\right. \\
& \left.\left.-\boldsymbol{J}_{T}^{T} \boldsymbol{\lambda}_{1}-c_{1} \boldsymbol{J}_{W}^{T}\left(\boldsymbol{J}_{W} \dot{q}-\dot{\boldsymbol{N}}_{1 d}\right)-\boldsymbol{J}_{W}^{T} \boldsymbol{\lambda}_{2}\right)\right) \\
\dot{\boldsymbol{\lambda}}_{1}= & k\left(\boldsymbol{J}_{T} \dot{\boldsymbol{q}}-\dot{\boldsymbol{X}}_{d}\right) \\
\dot{\boldsymbol{\lambda}}_{2}= & k\left(\boldsymbol{J}_{W} \dot{\boldsymbol{q}}-\dot{\boldsymbol{N}}_{1 d}\right)
\end{aligned}
$$

As already mentioned, this problem can be online solved using a RNN, as in [11], [26], whose weights can be directly computed using the system model. Moreover, as doing in this way the problem is solved iteratively, Section III-D.3 discusses the convergence of the algorithm to an equilibrium point that is the solution of optimization problem in (1).

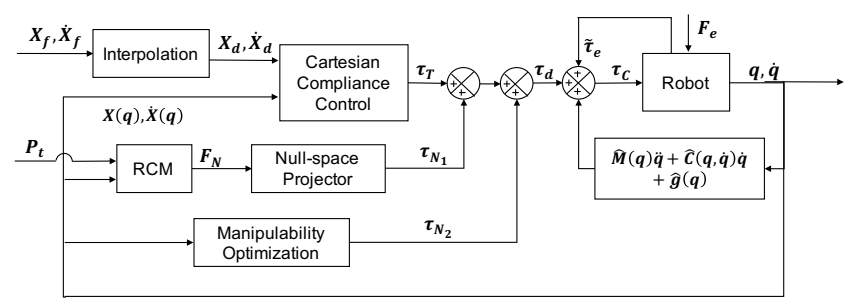

Fig. 5. Block diagram representing the proposed control architecture.

3) Convergence analysis: Letting $\boldsymbol{\Upsilon}=\left[\dot{\boldsymbol{q}}, \boldsymbol{\lambda}_{1}, \boldsymbol{\lambda}_{2}\right]^{T}$, the dynamical system can be rewritten as [11]:

$$
\dot{\boldsymbol{\Upsilon}}=-\boldsymbol{\Upsilon}+\boldsymbol{P}_{\Omega}[\boldsymbol{\Upsilon}-k \boldsymbol{F}(\boldsymbol{\Upsilon})]
$$

where $k$ is a constant, $\boldsymbol{P}_{\Omega}$ are the upper and lower limits of the joint velocities and the joint angles, and $\boldsymbol{F}(\mathbf{\Upsilon})=$ $\left[-\frac{\partial L}{\partial \dot{\boldsymbol{q}}}, \frac{\partial L}{\partial \boldsymbol{\lambda}_{1}}, \frac{\partial L}{\partial \boldsymbol{\lambda}_{2}}\right]^{T}$.

Given the expression of $\boldsymbol{F}(\mathbf{\Upsilon})$, the following relation can be derived:

$$
\nabla \boldsymbol{F}(\boldsymbol{\Upsilon})=\left[\begin{array}{ccc}
-\frac{\partial^{2} L}{\partial \dot{\boldsymbol{q}}^{2}} & -\frac{\partial^{2} L}{\partial \dot{\boldsymbol{q}} \partial \boldsymbol{\lambda}_{1}} & -\frac{\partial^{2} L}{\partial \dot{\boldsymbol{q}} \partial \boldsymbol{\lambda}_{2}} \\
\left(\frac{\partial^{2} L}{\partial \boldsymbol{\lambda}_{1} \partial \dot{\boldsymbol{q}}}\right)^{T} & \frac{\partial^{2} L}{\partial \boldsymbol{\lambda}_{1}^{2}} & \frac{\partial^{2} L}{\partial \boldsymbol{\lambda}_{1} \partial \boldsymbol{\lambda}_{2}} \\
\left(\frac{\partial^{2} L}{\partial \boldsymbol{\lambda}_{2} \partial \dot{\boldsymbol{q}}}\right)^{T} & \left(\frac{\partial^{2} L}{\partial \boldsymbol{\lambda}_{1} \partial \boldsymbol{\lambda}_{2}}\right)^{T} & \frac{\partial^{2} L}{\partial \boldsymbol{\lambda}_{2}^{2}}
\end{array}\right]
$$

Moreover, as $\nabla \boldsymbol{F}(\Upsilon)$ is an anti-symmetric matrix, the following relations hold:

$$
\begin{aligned}
\frac{\partial^{2} L}{\partial \boldsymbol{\lambda}_{1}^{2}}=\frac{\partial^{2} L}{\partial \boldsymbol{\lambda}_{2}^{2}}=\frac{\partial^{2} L}{\partial \boldsymbol{\lambda}_{1} \partial \boldsymbol{\lambda}_{2}}=\frac{\partial^{2} L}{\partial \boldsymbol{\lambda}_{2} \partial \boldsymbol{\lambda}_{1}} & = \\
\frac{\partial^{2} L}{\partial \dot{\boldsymbol{q}} \partial \boldsymbol{\lambda}_{1}} & =\frac{\partial^{2} L}{\partial \dot{\boldsymbol{q}} \partial \boldsymbol{\lambda}_{2}}=0
\end{aligned}
$$

As a consequence of the existence of $\nabla \boldsymbol{F}(\Upsilon), \boldsymbol{F}(\Upsilon)$ is continuously differentiable and

$$
\begin{aligned}
& \nabla \boldsymbol{F}(\mathbf{\Upsilon})+\nabla^{T} \boldsymbol{F}(\mathbf{\Upsilon})= \\
& {\left[\begin{array}{cccc}
-c_{0} \boldsymbol{J}_{T} \boldsymbol{J}_{T}^{T}-c_{1} \boldsymbol{J}_{W} \boldsymbol{J}_{W}^{T} & \mathbf{0} & \mathbf{0} \\
\mathbf{0} & \mathbf{0} & \mathbf{0} \\
\mathbf{0} & \mathbf{0} & \mathbf{0}
\end{array}\right] \leq \mathbf{0}}
\end{aligned}
$$

This proves the convergence of algorithm (2) to an equilibrium that is the optimal solution of the problem introduced in (1), and thus the stability of the inner loop. The controller can be formulated as:

$$
\begin{aligned}
\boldsymbol{\tau}_{N 2}=\left[\left(\boldsymbol{Z}(\boldsymbol{q}) \boldsymbol{M}(\boldsymbol{q}) \boldsymbol{Z}(\boldsymbol{q})^{T}\right) \boldsymbol{Z}(\boldsymbol{q}) \boldsymbol{M}(\boldsymbol{q})\right]^{T} \\
\\
\left(\boldsymbol{K}_{N 2} \ddot{\boldsymbol{q}}_{d}-\boldsymbol{D}_{N 2} \dot{\boldsymbol{q}}\right)
\end{aligned}
$$

where $\boldsymbol{Z}(\boldsymbol{q})$ is a full rank null-space base matrix proposed in [27]. The stability of the outer loop has been analysed in [17]. The proposed Manipulability Optimization Control 
(MOC), whose block diagram is depicted in Figure 5, can be expressed as:

$$
\boldsymbol{\tau}_{d}=\boldsymbol{\tau}_{T}+\boldsymbol{\tau}_{N 1}+\boldsymbol{\tau}_{N 2}
$$
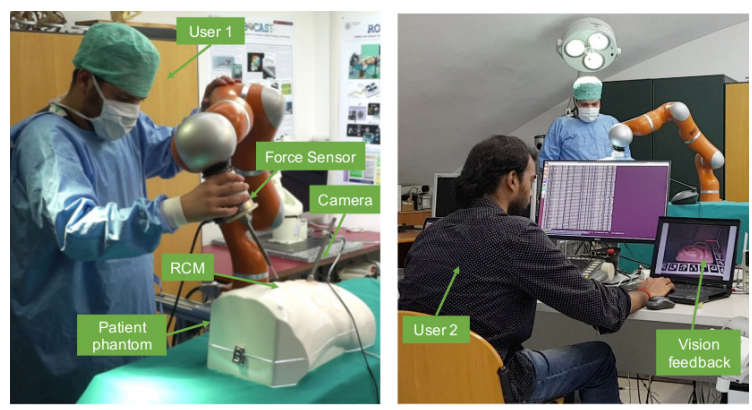

Fig. 6. Operative procedure: 1) hands-on control is activated to locate the trocar position (small incision on the patient's body); 2) autonomous tracking is used to control the tool tip along the virtual surgical tasks in augmented reality.

TABLE I

EXPERIMENTAL CONTROLLER PARAMETERS

\begin{tabular}{|c|l|}
\hline Controller & Controller parameters \\
\hline & $\boldsymbol{K}_{X}=\operatorname{diag}[2000,2000,2000]$ \\
DCAC & $\boldsymbol{D}_{X}=\operatorname{diag}[20,20,20]$ \\
$\left(\boldsymbol{\tau}_{d}=\boldsymbol{\tau}_{T}+\boldsymbol{\tau}_{N 1}\right)$ & $\boldsymbol{K}_{N}=\operatorname{diag}[600,600,600]$ \\
& $\boldsymbol{D}_{N}=\operatorname{diag}[10,10,10], \quad \lambda=0.5$ \\
\hline & $\boldsymbol{K}_{X}=\operatorname{diag}[3000,3000,3000]$ \\
& $\boldsymbol{D}_{X}=\operatorname{diag}[30,30,30]$ \\
MOC & $\boldsymbol{K}_{N}=\operatorname{diag}[800,800,800]$ \\
$\left(\boldsymbol{\tau}_{d}=\boldsymbol{\tau}_{T}+\boldsymbol{\tau}_{N 1}\right.$ & $\boldsymbol{D}_{N}=\operatorname{diag}[10,10,10], \quad \lambda=0.5$ \\
$\left.+\boldsymbol{\tau}_{N 2}\right)$ & $\boldsymbol{K}_{N 2}=[80,120,60,30,20,10,10]$ \\
& $\boldsymbol{K}_{D 2}=[6,10,5,3,2,1,1]$ \\
& $k_{0}=0.1$ \\
& $\boldsymbol{\lambda}_{\text {init }}=[0,0,0]^{T}, \boldsymbol{\lambda}_{2 \text { init }}=[0,0,0]^{T}$ \\
& $c_{0}=0.01, c_{1}=0.01, c_{2}=0.001, k=0.1$ \\
\hline
\end{tabular}

\section{EXPERIMENTAL RESULTS}

The set-up used to validate the proposed control algorithm is depicted in Figure 6. The operative procedure is organized as follows:

1) the medical staff locates the small incision on the patient's body;

2) the surgeon provides the desired trajectory $\boldsymbol{X}_{f}$ using the remote site to control the tool tip, and the desired trajectory $\boldsymbol{X}_{d}$ is generated by the control system using the interpolation function;

3) the robot autonomously tracks the desired trajectory $\boldsymbol{X}_{d}$ performing the surgical task.

During the last step, the impedance controller guarantees that the end-effector safely tracks the desired trajectory, the first null-space controller ensures that the RCM constraint is satisfied, and the second null-space controller optimizes the manipulability. The magnitude of the Cartesian position error $\left\|\boldsymbol{E}_{X}\right\|$, the RCM constraint error $d$ and the interaction force $\left\|\boldsymbol{F}_{e}\right\|$, defined in [20][21], are recorded to evaluate

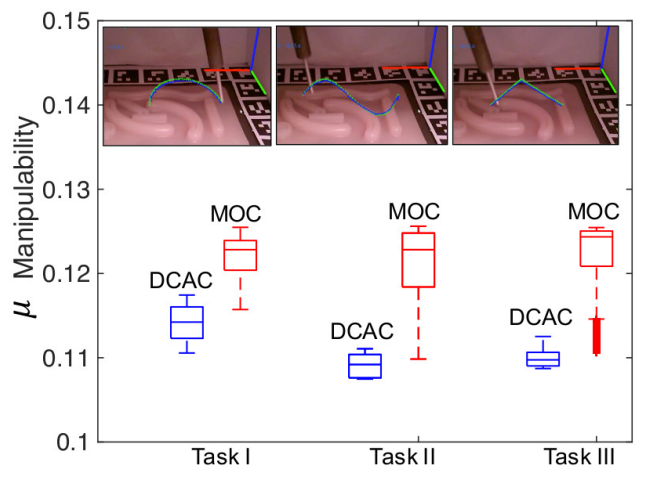

(a) Distribution of manipulability index $\mu$

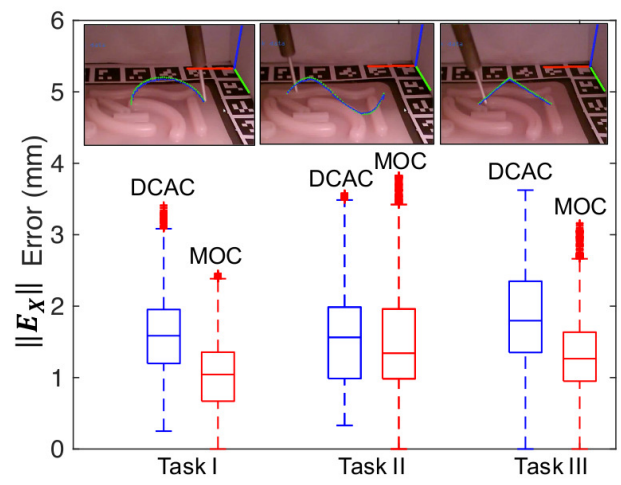

(b) Distribution of end-effector error $\left\|\boldsymbol{E}_{X}\right\|$

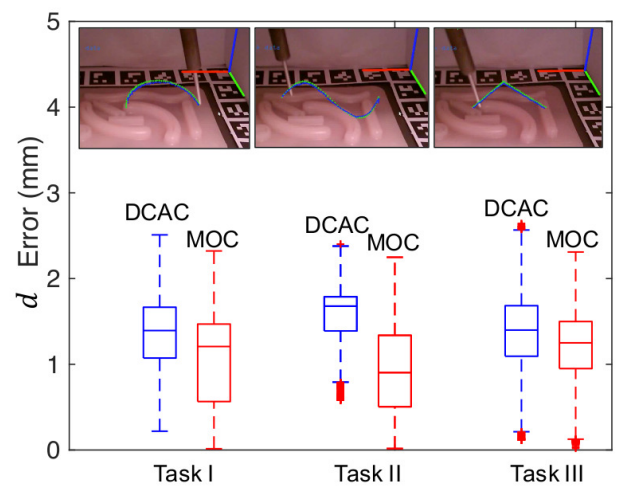

(c) Distribution of RCM constraint error $d$

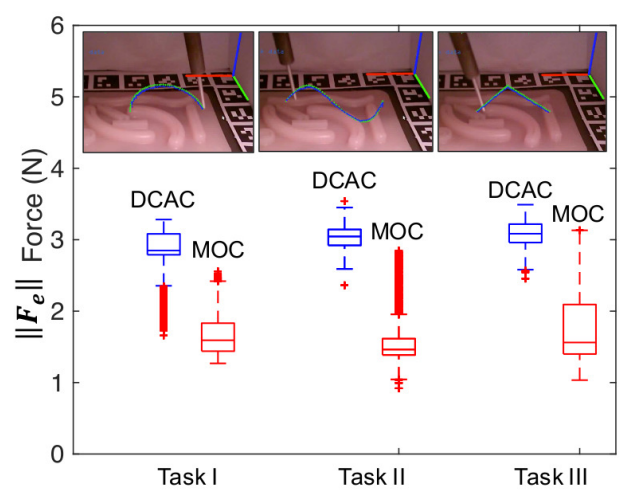

(d) Distribution of interaction force $\left\|\boldsymbol{F}_{e}\right\|$ on the RCM constraint

Fig. 7. Comparison between DCAC and MOC. 
the proposed control method.

The parameters of the controller can be found in Table I. The three virtual tasks [21] were performed with the Decoupled Cartesian Admittance Control (DCAC) [3] and with the proposed Manipulability Optimization Control (MOC) considering 5 trials for each algorithm.

Figure 7 shows the performance comparison between DCAC and MOC during autonomous tracking tasks. With the proposed algorithm, the manipulability index of the surgical tip has been increased over $7 \%$ with respect to DCAC. At the same time, the accuracy of the surgical tip has been improved, while the error of the RCM constraint have been decreased. Moreover, the interaction forces between the abdominal wall and the tool shaft are reduced. This demonstrates that the proposed MOC achieves better operational performance.

\section{CONCLUSION}

This paper proposes a novel manipulability optimization control of a serial redundant robot for online robot-assisted Minimally Invasive Surgery. A hierarchical operational space formulation [17] has been adopted, integrating three parts: a Cartesian compliance control strategy for the main surgical task, a first null-space controller to enforce the RCM constraint, and a second null-space controller for manipulability optimization by using constrained quadratic programming. Finally, an experimental evaluation has been performed on virtual surgical tasks, in order to validate the performance of the proposed methodology. Compared to [3], the results here reported demonstrate that the proposed control algorithm not only guarantees the RCM constraint during the autotracking phase, but also improves the manipulability of the robot. Achieving global manipulability optimization while guarantying the RCM constraint will be considered as a future work.

\section{REFERENCES}

[1] A. R. Lanfranco, A. E. Castellanos, J. P. Desai, and W. C. Meyers, "Robotic surgery: a current perspective," Annals of surgery, vol. 239, no. 1, p. 14, 2004.

[2] N. Aghakhani, M. Geravand, N. Shahriari, M. Vendittelli, and G. Oriolo, "Task control with remote center of motion constraint for minimally invasive robotic surgery," in Robotics and Automation (ICRA), 2013 IEEE International Conference on. IEEE, 2013, pp. 5807-5812.

[3] J. Sandoval, G. Poisson, and P. Vieyres, "Improved dynamic formulation for decoupled cartesian admittance control and rcm constraint," in Robotics and Automation (ICRA), 2016 IEEE International Conference on. IEEE, 2016, pp. 1124-1129.

[4] T. Ortmaier and G. Hirzinger, "Cartesian control issues for minimally invasive robot surgery," in Intelligent Robots and Systems, 2000.(IROS 2000). Proceedings. 2000 IEEE/RSJ International Conference on, vol. 1. IEEE, 2000, pp. 565-571.

[5] M. A. Peshkin, "Programmed compliance for error corrective assembly," IEEE Transactions on Robotics and Automation, vol. 6, no. 4, pp. 473-482, 1990.

[6] F. Petit, A. Dietrich, and A. Albu-Schäffer, "Generalizing torque control concepts: using well-established torque control methods on variable stiffness robots," IEEE Robotics and Automation Magazine, vol. 22 , no. 4 , pp. 37-51, 2015.

[7] P. J. From, "On the kinematics of robotic-assisted minimally invasive surgery," Modeling, Identification and Control, vol. 34, no. 2, p. 69, 2013.
[8] H. Su, G. Ferrigno, and E. De Momi, "Adaptive decoupling control of a serial redundant robot for teleoperated minimally invasive surgery," in IEEE ICRA Workshop on Supervised Autonomy in Surgical Robotics, 2018.

[9] M. D. Comparetti, E. De Momi, A. Vaccarella, M. Riechmann, and G. Ferrigno, "Optically tracked multi-robot system for keyhole neurosurgery," in Robotics and Automation (ICRA), 2011 IEEE International Conference on. IEEE, 2011, pp. 661-666.

[10] H. Su, N. Enayati, L. Vantadori, A. Spinoglio, G. Ferrigno, and E. De Momi, "Online human-like redundancy optimization for teleoperated anthropomorphic manipulators," International Journal of Advanced Robotic Systems, vol. 15, no. 6, p. 1729881418814695 , 2018.

[11] L. Jin, S. Li, H. M. La, and X. Luo, "Manipulability optimization of redundant manipulators using dynamic neural networks," IEEE Transactions on Industrial Electronics, 2017.

[12] T. M. Sobh and D. Y. Toundykov, "Optimizing the tasks at hand [robotic manipulators]," IEEE robotics \& automation magazine, vol. 11, no. 2, pp. 78-85, 2004.

[13] N. Vahrenkamp, T. Asfour, G. Metta, G. Sandini, and R. Dillmann, "Manipulability analysis." in Humanoids, 2012, pp. 568-573.

[14] B. Siciliano, "Kinematic control of redundant robot manipulators: A tutorial," Journal of intelligent and robotic systems, vol. 3, no. 3, pp. 201-212, 1990.

[15] R. V. Dubey, J. A. Euler, and S. M. Babcock, "Real-time implementation of an optimization scheme for seven-degree-of-freedom redundant manipulators," IEEE Transactions on Robotics and Automation, vol. 7, no. 5, pp. 579-588, 1991.

[16] H.-L. Wang, J.-W. Li, and H. Liu, "Practical limitations of an algorithm for the singular value decomposition as applied to redundant manipulators," in Robotics, Automation and Mechatronics, 2006 IEEE Conference on. IEEE, 2006, pp. 1-6.

[17] A. Dietrich, C. Ott, and J. Park, "The hierarchical operational space formulation: Stability analysis for the regulation case," IEEE Robotics and Automation Letters, vol. 3, no. 2, pp. 1120-1127, 2018.

[18] G. Schreiber, A. Stemmer, and R. Bischoff, "The fast research interface for the kuka lightweight robot," in IEEE Workshop on Innovative Robot Control Architectures for Demanding (Research) Applications How to Modify and Enhance Commercial Controllers (ICRA 2010), 2010, pp. 15-21.

[19] S. Garrido-Jurado, R. Muñoz-Salinas, F. J. Madrid-Cuevas, and M. J. Marín-Jiménez, "Automatic generation and detection of highly reliable fiducial markers under occlusion," Pattern Recognition, vol. 47, no. 6, pp. 2280-2292, 2014.

[20] H. Su, J. Sandoval, M. Makhdoomi, G. Ferrigno, and E. De Momi, "Safety-enhanced human-robot interaction control of redundant robot for teleoperated minimally invasive surgery," in 2018 IEEE International Conference on Robotics and Automation (ICRA). IEEE, 2018, pp. 6611-6616.

[21] H. Su, C. Yang, G. Ferrigno, and E. De Momi, "Improved human-robot collaborative control of redundant robot for teleoperated minimally invasive surgery," IEEE Robotics and Automation Letters, vol. 4, no. 2, pp. 1447-1453, 2019.

[22] C. Ott, "Cartesian impedance control: The rigid body case," in Cartesian Impedance Control of Redundant and Flexible-Joint Robots. Springer, 2008, pp. 29-44.

[23] A. Dietrich, Whole-body impedance control of wheeled humanoid robots. Springer, 2016.

[24] H. Su, J. Sandoval, P. Vieyres, G. Poisson, G. Ferrigno, and E. De Momi, "Safety-enhanced collaborative framework for teleoperated minimally invasive surgery using a 7-dof torque-controlled robot," International Journal of Control, Automation and Systems, vol. 16, no. 6, pp. 2915-2923, 2018.

[25] J. Sandoval, H. Su, P. Vieyres, G. Poisson, G. Ferrigno, and E. De Momi, "Collaborative framework for robot-assisted minimally invasive surgery using a 7-dof anthropomorphic robot," Robotics and Autonomous Systems, vol. 106, pp. 95-106, 2018.

[26] Y. Li, S. Li, and B. Hannaford, "A novel recurrent neural network for improving redundant manipulator motion planning completeness," in International Conference on Robotics and Automation, 2018, pp. 2956-2961.

[27] M. Z. Huang and H. Varma, "Optimal rate allocation in kinematicallyredundant manipulators-the dual projection method," in Robotics and Automation, 1991. Proceedings., 1991 IEEE International Conference on. IEEE, 1991, pp. 702-707. 